\title{
Thermodynamics of solitonic matter waves in a toroidal trap
}

\author{
L. Salasnich, ${ }^{1}$ A. Parola, ${ }^{2}$ and L. Reatto ${ }^{3}$ \\ ${ }^{1}$ CNR-INFM and CNISM, Unità di Milano Università, Via Celoria 16, 20133 Milano, Italy \\ ${ }^{2}$ Dipartimento di Fisica e Matematica, Università dell'Insubria, Via Valleggio 11, 22100 Como, Italy \\ ${ }^{3}$ Dipartimento di Fisica and CNISM, Università di Milano, Via Celoria 16, 20133 Milano, Italy \\ (Received 13 April 2006; published 19 September 2006)
}

\begin{abstract}
We investigate the thermodynamic properties of a Bose-Einstein condensate with negative scattering length confined in a toroidal trapping potential. By numerically solving the coupled Gross-Pitaevskii and Bogoliubov-de Gennes equations, we study the phase transition from the uniform state to the symmetrybreaking state characterized by a bright-soliton condensate and a localized thermal cloud. In the localized regime, three states with a finite condensate fraction are present: the thermodynamically stable localized state, a metastable localized state, and also a metastable uniform state. Remarkably, the presence of the stable localized state strongly increases the critical temperature of Bose-Einstein condensation.
\end{abstract}

In recent experiments, a repulsive Bose-Einstein condensate (BEC) has been produced and studied in a quasi-onedimensional (1D) ring [1,2]. These experiments and also previous experimental investigations with cold atoms in torodial traps [3] are important steps to achieve ultrahighprecision sensors with atom interferometry (see, for instance, Ref. [4]). The case of an attractive BEC in a ring has not yet been experimentally investigated but appears very interesting: a quantum phase transition from an azimuthally uniform condensate to a bright soliton has been predicted $[5,6]$. Dynamically stable multipeak bright solitons may also appear [7].

In this paper, we investigate the effect of temperature on the uniform-to-localized phase transition by solving the Gross-Pitaevskii equation (GPE) of the macroscopic wave function of the Bose condensate and the Bogoliubov-de Gennes equations for the quantum depletion and the thermal cloud. We show that, for a fixed number $N$ of atoms, the uniform solution always exists but it is thermodynamically stable only up to a critical interaction strength that depends on the temperature. Above this critical interaction strength, both the condensate and the thermal cloud become localized. In addition, we determine the finite-temperature phase diagram of the system for a sample of alkali-metal atoms in a ring. Our results are relevant not only for the physics of cold atoms but also for the nonlinear science of solitons. In fact, a mixture of condensed and noncondensed attractive particles [8] correspond to incoherent solitons of light in Kerr media [9].

We consider a Bose gas with negative scattering length $\left(a_{s}<0\right)$ confined in a toroidal potential and model the transverse confinement with a harmonic potential of frequency $\omega_{\perp}$. The two characteristic lengths of the toroidal trap are the azimuthal radius $R$ and the transverse harmonic length $a_{\perp}=\left[\hbar /\left(m \omega_{\perp}\right)\right]^{1 / 2}$. To avoid the confinement-induced resonance at $\left|a_{s}\right| \simeq a_{\perp}[10]$, we impose that $\left|a_{s}\right| \ll a_{\perp}$ [11]. At low temperature $T$ and with a finite number $N$ of atoms, in the Bose gas there is the thermal cloud but also the BEC. Under the condition $R \gg a_{\perp}$, the azimuthal wave function $\phi(z)$ of the BEC in the ring satisfies the 1D nonpolynomial Schrödinger equation (NPSE) [7,12],

$$
\left[-\frac{\hbar^{2}}{2 m} \partial_{z}^{2}+\mu\left(n_{0}(z)\right)\right] \phi(z)=\bar{\mu} \phi(z),
$$

where $z=R \theta$ is the azimuthal coordinate with $\theta$ the azimuthal angle. This equation has been deduced from the 3D GPE by using a Gaussian transverse wave function with a densitydependent width and neglecting curvature effects [7,12]. The nonpolynomial term $\mu\left(n_{0}(z)\right)$ is a function of the BEC density $n_{0}(z)=N_{0}|\phi(z)|^{2}$ and it is given by $\mu=\partial \mathcal{E} / \partial n_{0}$, where $\mathcal{E}=\hbar \omega_{\perp} n_{0}\left(1+2 a_{s} n_{0}\right)^{1 / 2}$ and $a_{s}<0$ is the 3D $s$-wave scattering length [7]. The wave function $\phi(z)$ is normalized to 1 and satisfies the condition of periodicity $\phi(z+L)=\phi(z)$, where $L=2 \pi R$. The chemical potential $\bar{\mu}$ is fixed by the normalization condition $\int|\phi(z)|^{2} d z=1$. Quantum and thermal depletions of the BEC are obtained by solving the Bogoliubov-de Gennes (BdG) equations [13] for the quasiparticle amplitudes $u_{\alpha}(z)$ and $v_{\alpha}(z)$, given by

$$
\mathcal{L}\left(\begin{array}{l}
u_{\alpha}(z) \\
v_{\alpha}(z)
\end{array}\right)=\epsilon_{\alpha}\left(\begin{array}{l}
u_{\alpha}(z) \\
v_{\alpha}(z)
\end{array}\right),
$$

where $\epsilon_{\alpha}$ are the energies of quasiparticle excitations and the operator $\mathcal{L}$ is

$$
\mathcal{L}=\left(\begin{array}{cc}
-\frac{\hbar^{2}}{2 m} \partial_{z}^{2}-\bar{\mu}+\frac{\partial\left(n_{0} \mu\right)}{\partial n_{0}} & N_{0} \phi^{2} \frac{\partial \mu}{\partial n_{0}} \\
-N_{0}\left(\phi^{*}\right)^{2} \frac{\partial \mu}{\partial n_{0}} & \frac{\hbar^{2}}{2 m} \partial_{z}^{2}+\bar{\mu}-\frac{\partial\left(n_{0} \mu\right)}{\partial n_{0}}
\end{array}\right)
$$

The local density of the quantum depletion reads $n_{\text {out }}(z)=\Sigma_{\alpha}\left|v_{\alpha}(z)\right|^{2}$, while the thermal local density is $n_{T}(z)$ $=\Sigma_{\alpha}\left[\left|u_{\alpha}(z)\right|^{2}+\left|v_{\alpha}(z)\right|^{2}\right] \bar{n}_{\alpha}$, where $\bar{n}_{\alpha}=\left[\exp \left(\epsilon_{\alpha} / k_{B} T\right)-1\right]^{-1}$ are the Bose numbers of occupation for the noninteracting quasiparticles, $k_{B}$ is the Boltzmann constant, and $T$ is the absolute temperature of the thermal cloud. The number $\tilde{N}$ of noncondensed atoms is thus given by $\tilde{N}=N_{\text {out }}+N_{T}$, where $N_{\text {out }}=\int n_{\text {out }}(z) d z$ and $N_{T}=\int n_{T}(z) d z$. In the weak-coupling limit, where $a_{s} n_{0} \ll 1$, the energy density $\mathcal{E}$ becomes $\mathcal{E}=\hbar \omega_{\perp} n_{0}+\hbar \omega_{\perp} a_{s} n_{0}^{2}$, the NPSE (1) reduces to the familiar 
1D GPE [7], and the BdG equations (2) and (3) reduce to the BdG equations of the strictly 1D problem. Note that Eqs. (1)-(3) could be improved by taking into account Popov and Beliaev corrections, which are usually not negligible only when the condensed fraction is very small [14].

In our approach, by fixing the total number $N=N_{0}+\tilde{N}$ of atoms and the temperature $T$, the equilibrium configurations are found from the Helmholtz free energy [13],

$$
F=E_{0}+\widetilde{E}+\bar{\mu}\left(N-N_{0}\right)-T S,
$$

where

$$
E_{0}=N_{0} \int\left[-\frac{\hbar^{2}}{2 m} \phi^{*}(z) \partial_{z}^{2} \phi(z)+\mathcal{E}\left(n_{0}(z)\right)\right] d z
$$

is the BEC energy. The out-of-condensate energy $\widetilde{E}$ and entropy $S$ are instead given by

$$
\begin{gathered}
\widetilde{E}=\sum_{\alpha} \epsilon_{\alpha}\left[\bar{n}_{\alpha}-\int\left|v_{\alpha}(z)\right|^{2} d z\right], \\
S=-k_{B} \sum_{\alpha}\left[\bar{n}_{\alpha} \log \left(\bar{n}_{\alpha}\right)-\left(1+\bar{n}_{\alpha}\right) \log \left(1+\bar{n}_{\alpha}\right)\right] .
\end{gathered}
$$

The solution of the 1D GPE with periodic boundary conditions is known analytically [5]: it is uniform for $0<\gamma N_{0}<\pi^{2} a_{\perp} / L$, with $\gamma=2\left|a_{s}\right| / a_{\perp}$ the interatomic strength, and becomes localized for $\gamma N_{0}>\pi^{2} a_{\perp} / L$. By using the NPSE, we find that the transition strength approaches the 1D GPE one for large $L[7]$. The main difference between 1D GPE and NPSE is that the NPSE correctly gives the collapse of the localized solution for $\gamma N_{0}>4 / 3$, while the 1D GPE does not predict collapse [7]. It is important to observe that the uniform-to-localized transition depends on $N_{0}$ and only implicitly on $N=N_{0}+N_{\text {out }}+N_{T}$. We shall show that, for a fixed number $N$ of atoms, the uniform solution exists for any value of the interatomic strength $\gamma$ but it is thermodynamically stable only below a critical strength $\gamma_{c}$. Above $\gamma_{c}$, the stable state is a localized solution that minimizes the free energy.

The BdG equations (2) and (3) are easily solved if $\phi(z)$ is uniform: $\phi(z)=1 / \sqrt{L}$. In this case, the quasiparticle amplitudes $u_{k}(z)=\bar{u}_{k} e^{i k z}$ and $v_{k}(z)=\bar{v}_{k} e^{-i k z}$ are plane waves and the wave vector $k$ is quantized: $k=(2 \pi / L) j$, with $j$ an integer number. When $\phi(z)$ is not uniform, one must numerically solve Eqs. (1)-(3). We use a finite-difference space discretization and diagonalize the matrix associated to the operator $\mathcal{L}$ of Eq. (3). In our calculations, we use various matrix dimensions up to $4000 \times 4000$. The numerical procedure is discussed in Ref. [15]. In our approach, the transverse excitations do not contribute to the thermodynamics: this assumption is reasonable only if the system is quasi-1D.

In Fig. 1, we plot the results of our numerical calculations of the free energy $F$ as a function of the scaled interatomic strength $\gamma=2\left|a_{s}\right| / a_{\perp}$ for increasing values of the temperature $T$. We choose $N=10^{4}$ atoms and a toroidal geometry with $L / a_{\perp}=100$. While the uniform solution (dashed line) exists for any strength $\gamma$, the localized solution (solid line) exists only above a critical strength $\gamma_{e}$. As expected, at

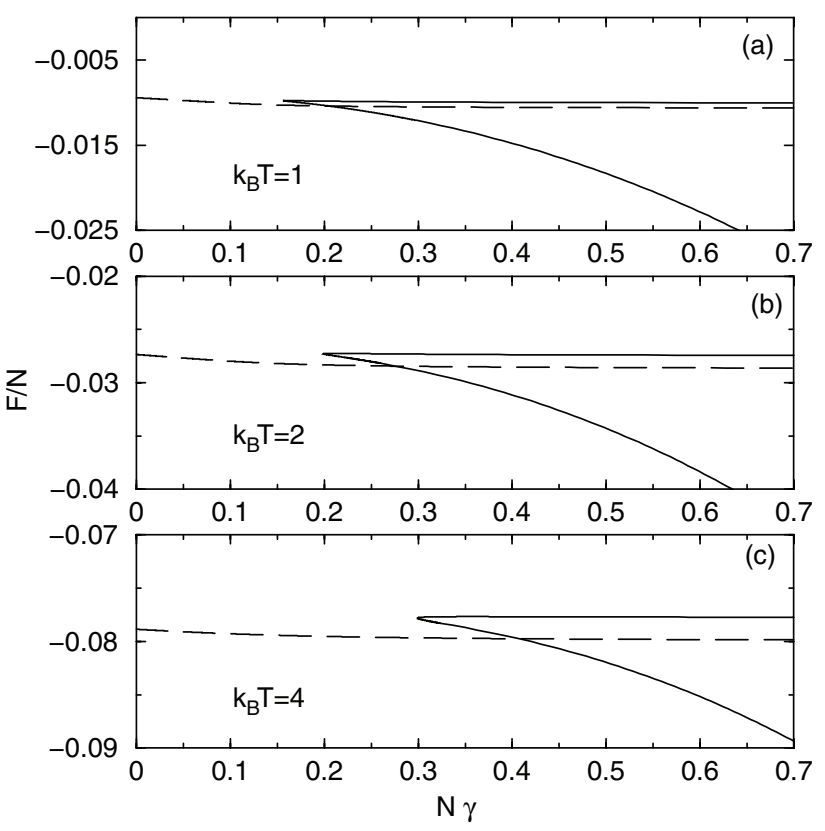

FIG. 1. Free energy $F$ as a function of the scaled interatomic strength $\gamma=2\left|a_{s}\right| / a_{\perp}$ for an attractive Bose gas of $N=10^{4}$ atoms in a toroidal trap with $L / a_{\perp}=100$. Dashed line: uniform solution. Solid line: localized solutions. The energies $F$ and $k_{B} T$ are in units of $\hbar \omega_{\perp}$.

$T \simeq 0$ we find $\gamma_{c} \simeq \pi^{2} a_{\perp} / L=0.0987$. For a given $\gamma\left(\gamma>\gamma_{e}\right)$, there are two localized states: one is thermodynamically stable (lower solid line) and the other is metastable (upper solid line). The metastability is guaranteed by the Bogoliubov eigenvalues being real. At very low temperature, see panel (a) of Fig. 1, for $\gamma>\gamma_{e}$ the solid line of the metastable localized solution is very close to the dashed line of the metastable uniform solution. At higher temperature, see panels (b) and (c) of Fig. 1, the free energy of the metastable localized solution, but also the free energy of the stable one at small $\gamma>\gamma_{e}$, is higher than the free energy of the uniform solution. The strength $\gamma_{c}$ at which the dashed line crosses the lower solid line determines the point where the first-order transition takes place. At $T \simeq 0$, we find that $\gamma_{c} \simeq \gamma_{e}$. The strength $\gamma_{c}$ grows with the temperature $T$, but in general $\gamma_{c}$ does not coincide with the strength $\gamma_{e}$ at which the localized solutions appear. Thus, at finite temperature, for $0<\gamma<\gamma_{e}$ there is only the stable uniform state; for $\gamma_{e}<\gamma<\gamma_{c}$, there are two metastable localized states and the stable uniform state; for $\gamma>\gamma_{c}$, the stable state is one of the two localized states while the other localized state and the uniform state are metastable.

A coherent bright soliton and a thermal cloud coexist in the stable state, as in the metastable localized state. This is clearly illustrated in Fig. 2, where we plot the condensate fraction $N_{0} / N$ as a function of $\gamma$ for different temperatures. At low temperature, see panel (a) of Fig. 2, the uniform state (dashed line) is a quasipure BEC for $0<\gamma<\gamma_{e}$. For $\gamma>\gamma_{e}$, the condensate fraction of the uniform state quickly decreases. At low temperature, the uniform-to-localized phase transition does not produce a relevant quantum depletion $N_{\text {out }} / N$ at the transition point. This result is obtained with a 


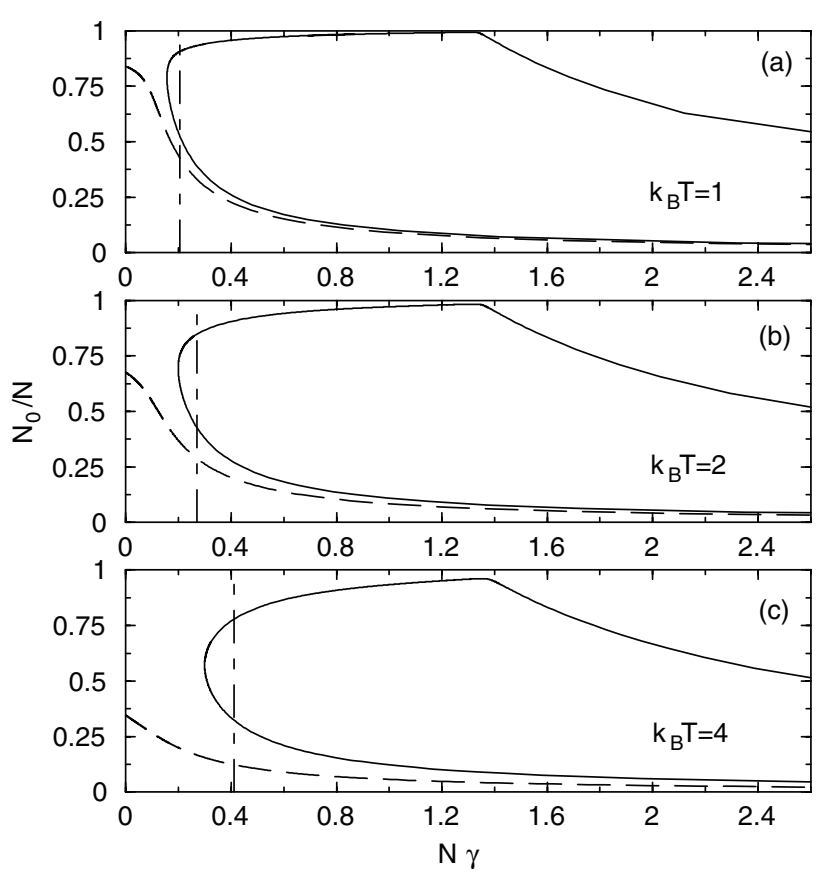

FIG. 2. Condensate fraction $N_{0} / N$ as a function of the scaled interatomic strength $\gamma=2\left|a_{s}\right| / a_{\perp}$ for $N=10^{4}$ atoms with $L / a_{\perp}=100$. Dashed line: uniform solution. Solid line: localized solutions. The vertical dot-dashed line indicates the strength $N \gamma_{c}$ at which the uniform-to-localized phase transition takes place.

fixed number of atoms $\left(N=10^{4}\right)$. We have verified that by fixing $N_{0}$ instead of $N$ (as done in Ref. [5]), the condensate fraction $N_{0} / N$ goes to zero for $\gamma N_{0} \simeq \pi^{2} a_{\perp} / L$.

At higher temperature, see panels (b) and (c) of Fig. 2, BEC and noncondensate components coexist also in the uniform state. Figure 2 shows that the condensate fraction of the localized states is higher than the condensate fraction of the uniform state. Remarkably, at the transition strength $\gamma_{c}$ there is a sizeable jump in the condensate fraction. Moreover, for the stable localized state, the ratio $N_{0} / N$ initially grows by increasing the interatomic strength. As discussed previously, the BEC soliton of the localized state will collapse at $\gamma N_{0} \simeq 4 / 3$, but the true collapse is avoided by populating the out-of-condensate cloud with $\tilde{N}=N-N_{0}$ atoms for $N \gamma>4 / 3$. We have verified that in the stable localized state, the number $N_{0}$ of condensed atoms is always very close to the collapse value $4 /(3 \gamma)$ and, for a fixed $\gamma$, the number $N_{0}$ has a very small temperature dependence. It follows that one can estimate the condensed fraction of the stable localized state as $N_{0} / N \simeq 4 /(3 N \gamma)$. This estimation is not so rough: choosing, for instance, $N \gamma=2$, one finds $N_{0} / N \simeq 2 / 3$, in good agreement with the result shown in the upper solid line of Fig. 2 (see where $N_{0} / N=0.66$ ).

In the previous calculations, transverse modes have been neglected and the thermally excited quasiparticles are allowed to populate only the lowest transverse state. This assumption can be justified by a simple argument: In the weakly localized regime (low $N \gamma$ ), the spectrum of elementary excitations is well approximated by the free Bose gas, where the longitudinal modes have energies $O\left(\hbar \omega_{\perp} a_{\perp}^{2} / L^{2}\right)$

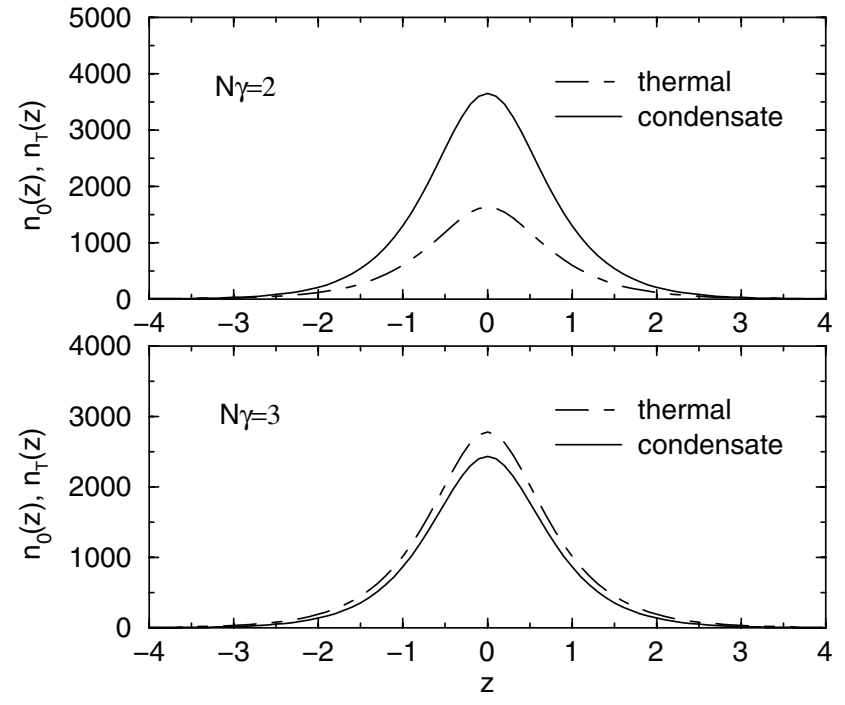

FIG. 3. Azimuthal density profiles of the thermodynamically stable state for $N=10^{4}$ atoms with $L / a_{\perp}=100$ and $k_{B} T /\left(\hbar \omega_{\perp}\right)=2$. Length $z$ is in units of $a_{\perp}=\left[\hbar /\left(m \omega_{\perp}\right)\right]^{1 / 2}$.

much lower than the $O\left(\hbar \omega_{\perp}\right)$ transverse modes. In this limit, we have explicitly verified that, with the chosen parameters $\left(L / a_{\perp}=100\right)$, for $k_{B} T \lesssim 5 \hbar \omega_{\perp}$ the number of quasiparticles in the excited transverse modes is indeed negligible. In the strongly localized region, both longitudinal and transverse low-energy excitations are known in the large- $L$ limit $[6,17]$, where a finite $O\left(\hbar \omega_{\perp}\right)$ gap separates the two branches. Moreover, our calculations show that for $N \gamma<4 / 3$, thermal depletion does not affect deeply the thermodynamics of the model up to the transition temperature.

For completeness, we have repeated the calculations by using the 1D GPE. Also in this case we have found two localized solutions, but the stable one always maintains a condensate fraction close to 1 , due to the fact that the 1D GPE does not predict the collapse. Thus the transverse structure of the soliton, taken into account by the NPSE, strongly modifies the thermodynamics of the attractive Bose gas.

In Fig. 3, we plot the density profile $n_{0}(z)$ of the condensate cloud (solid line) and of the density profile $n_{T}(z)$ of the thermal cloud (dot-dashed line) for the stable localized state and two values of $\gamma$. We have verified that the density profile $n_{\text {out }}(z)$ of the quantum depletion reduces by increasing the temperature $T$, and that the quantum depletion $N_{\text {out }} / N$ becomes relevant only when $k_{B} T$ is of the order of the lowest Bogoliubov energy level. This energy can be easily estimated as $k_{B} T /\left(\hbar \omega_{\perp}\right)=\left[\hbar^{2} /(2 m)\right](2 \pi / L)^{2} /\left(\hbar \omega_{\perp}\right)=2 \pi^{2} a_{\perp}^{2} / L^{2} \simeq 0.002$.

In Fig. 4, we plot the phase diagram of the atomic cloud of bosons in the plane $(N \gamma, T)$ for $L / a_{\perp}=100$ and $N=10^{4}$. In the figure, we insert a dashed line at $k_{B} T /\left(\hbar \omega_{\perp}\right)=6.01$, which is the transition temperature $T_{\mathrm{BEC}}$ of the Bose-Einstein condensation above which the condensate fraction of the uniform state is zero. The transition temperature $T_{\mathrm{BEC}}$ reduces by increasing $L / a_{\perp}$. In fact, it is easy to show [16] that $k_{B} T_{\mathrm{BEC}} /\left(\hbar \omega_{\perp}\right)$ scales as $N a_{\perp}^{2} / L^{2}$. Figure 4 displays as a solid line the curve of the uniform-to-localized transition. Remark- 


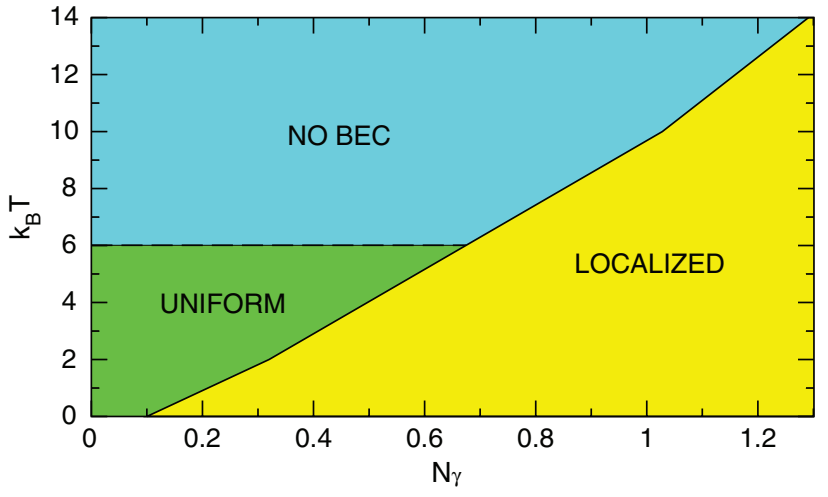

FIG. 4. (Color online) Phase diagram in the plane temperature $T$ vs interatomic strength $\gamma$ for $N=10^{4}$ atoms in a toroidal trap, with $L=100$. The energy $k_{B} T$ is in units of $\hbar \omega_{\perp}$ and the length in units of $a_{\perp}$.

ably, the BEC transition temperature of the localized solution is much larger than the uniform one. Figure 4 shows that with $N \gamma>0.68$, there is a first-order transition from the localized BEC phase to the uniform thermal phase without BEC. Instead, for $N \gamma<0.68$ the no-BEC phase is obtained starting from the uniform phase by increasing the temperature.

In conclusion, we have shown that an attractive Bose condensate in a quasi-one-dimensional toroidal trap displays at finite temperature new and interesting features, such as the coexistence of a coherent self-bound bright soliton with a thermal cloud, the avoidance of true collapse via population of the out-of-condensate (thermal) component, and the enhancement of the Bose-Einstein transition temperature for the localized solution. We stress that our predictions can be verified by using a magnetic ring with geometric parameters not too far from those used in Ref. [1] for trapping a repulsive condensate of ${ }^{87} \mathrm{Rb}$ atoms. For instance, a sample of ${ }^{7} \mathrm{Li}$ atoms, which have a negative scattering length $\left(a_{s}=-1.4 \mathrm{~nm}\right)$, can be trapped in a ring with transverse width $a_{\perp} \simeq 6 \mu \mathrm{m}$, which gives $\hbar \omega_{\perp} / k_{B}=\hbar^{2} /\left(2 a_{\perp}^{2}\right) \simeq 2 \mathrm{nK}$. In this way, having $N=10^{4}$ atoms, the interaction strength is $N \gamma=N 2\left|a_{s}\right| / a_{\perp} \simeq 5$. Then, by taking the azimuthal radius $R=L /(2 \pi) \simeq 100 \mu \mathrm{m}$, one can test the predictions of Fig. 4 varying the temperature or tuning the scattering length $a_{s}$ around a Feshbach resonance.
[1] S. Gupta, K. W. Murch, K. L. Moore, T. P. Purdy, and D. M. Stamper-Kurn, Phys. Rev. Lett. 95, 143201 (2005).

[2] A. S. Arnold, C. S. Garvie, and E. Riis, e-print cond-mat/ 0506142.

[3] J. A. Sauer, M. D. Barrett, and M. S. Chapman, Phys. Rev. Lett. 87, 270401 (2001); A. Kasper et al., J. Opt. B: Quantum Semiclassical Opt. 5, S143 (2003); A. Hopkins, B. Lev, and H. Mabuchi, Phys. Rev. A 70, 053616 (2004).

[4] M. A. Kasevich, Science 298, 1363 (2002).

[5] G. M. Kavoulakis, Phys. Rev. A 67, 011601(R) (2003); R. Kanamoto, H. Saito, and M. Ueda, ibid. 67, 013608 (2003).

[6] R. Kanamoto, H. Saito, and M. Ueda, Phys. Rev. A 68, 043619 (2003); G. M. Kavoulakis, ibid. 69, 023613 (2004).

[7] A. Parola, L. Salasnich, R. Rota, and L. Reatto, Phys. Rev. A 72, 063612 (2005).

[8] H. Buljan, M. Segev, and A. Vardi, Phys. Rev. Lett. 95, 180401 (2005); B. Pozzi, L. Salasnich, A. Parola, and L. Reatto, J. Low Temp. Phys. 119, 57 (2000).

[9] M. Mitchell and M. Segev, Nature (London) 387, 880 (1997);
M. Mitchell, M. Segev, T. H. Coskun, and D. N. Christodoulides, Phys. Rev. Lett. 79, 4990 (1997).

[10] M. Olshanii, Phys. Rev. Lett. 81, 938 (1998).

[11] L. Salasnich, A. Parola, and L. Reatto, Phys. Rev. A 70, 013606 (2004); 72, 025602 (2005).

[12] L. Salasnich, A. Parola, and L. Reatto, Phys. Rev. A 65, 043614 (2002).

[13] A. L. Fetter, in Bose Einstein Condensation in Atomic Gases, Proceedings of the Enrico Fermi School, Course CXL, edited by M. Inguscio, S. Stringari, and C. W. Wieman (SIF, Bologna, 1999).

[14] A. Griffin, Phys. Rev. B 53, 9341 (1996); H. Shi and A. Griffin, Phys. Rep. 304, 1 (1998).

[15] L. Salasnich, A. Parola, and L. Reatto, Phys. Rev. A 60, 4171 (1999).

[16] V. I. Yukalov, Phys. Rev. A 72, 033608 (2005).

[17] L. Salasnich, A. Parola, and L. Reatto, Phys. Rev. A 66, 043603 (2002). 